\title{
Multi-scale Rule-of-Mixtures Model of Carbon Nanotube/Carbon Fiber/Epoxy Lamina
}

\author{
S. J. V. Frankland ${ }^{1}$, J. C. Riddick ${ }^{2}$ and T. S. Gates ${ }^{3}$ \\ ${ }^{1}$ National Institute of Aerospace, 100 Exploration Way, Hampton, VA 23666, U. S. A. \\ ${ }^{2}$ Vehicle Technology Directorate, US Army Research Laboratory, Hampton, VA 23681, \\ U.S.A. \\ ${ }^{3}$ Mechanics of Structures and Materials Branch, NASA Langley Research Center, Hampton, \\ VA 23681, U. S. A.
}

\begin{abstract}
A unidirectional carbon fiber/epoxy lamina in which the carbon fibers are coated with single-walled carbon nanotubes is modeled with a multi-scale method, the atomistically informed rule-of-mixtures. This multi-scale model is designed to include the effect of the carbon nanotubes on the constitutive properties of the lamina. It included concepts from the molecular dynamics/equivalent continuum methods, micromechanics, and the strength of materials. Within the model both the nanotube volume fraction and nanotube distribution were varied. It was found that for a lamina with $60 \%$ carbon fiber volume fraction, the Young's modulus in the fiber direction varied with changes in the nanotube distribution, from 138.8 to $140 \mathrm{GPa}$ with nanotube volume fractions ranging from 0.0001 to 0.0125 . The presence of nanotube near the surface of the carbon fiber is therefore expected to have a small, but positive, effect on the constitutive properties of the lamina.
\end{abstract}

\section{INTRODUCTION}

Near term applications of carbon nanotubes (CNT) in materials for aerospace vehicles are most likely to be realized with mechanical or electrical applications that require small quantities of nanotubes. To implement carbon nanotubes as structural members could be accomplished by the selective use of carbon nanotubes in critical areas of traditional composites. For example, carbon nanotubes added between the layers of a traditional carbon fiber/epoxy laminate may well improve the interface strength between carbon fiber and epoxy matrix, and thereby improve fracture toughness of these laminates.

The effectiveness of adding carbon nanotubes to laminates can be analyzed with multi-scale models which are capable of incorporating atomistic details. At the atomisticlevel the assumptions of 'perfect' bonding between the various contributors to the laminate can be removed in detailed atomistic simulations. With properties at this level established, the micro-scale properties can be accessed with more standard micromechanics methods such as the Mori-Tanaka method or the rule-of-mixtures analysis[1]. Multi-scale models have applied atomistic simulation and micromechanics to the constitutive properties of various functionalized nanotube materials [2-5]. There are some atomistic simulations of epoxy/nanotube composites by other researchers which address nanotube pull-out [6]. Also, a more recent study on nanotubes chemically bonded into the epoxy matrix showed a 
Young's modulus of up to $160 \mathrm{GPa}$ in the direction of the nanotube axis, and 4-8 GPa in the transverse direction at a nanotube volume fraction of $25 \%$ [7].

In the present work, the constitutive properties of a unidirectional carbon fiber/epoxy lamina are modeled by a multi-scale model. In the lamina, each carbon fiber is uniformly coated with carbon nanotubes. The objective is to calculate the Young's modulus in the direction of the carbon fiber of unidirectionally-reinforced carbon fiber/epoxy laminae, where the nanotube distribution around the fiber is varied. For this purpose, we develop the atomistically informed rule-of-mixtures approach. This method requires a multi-scale approach to the analysis of several components of the nanotube-coated carbon fiber. The constitutive properties of the nanoscale components are modeled with the molecular dynamics/equivalent continuum (MD/EC) model. The CNT distribution and volume are modeled using the $\mathrm{MD} / \mathrm{EC}$ model in conjunction with the Mori-Tanaka micromechanics method to calculate the Young's modulus over a defined nanotube distribution. A description of each stage of the multi-scale model is presented, and the results of applying the atomistically-informed rule-of-mixtures are given for a lamina with $60 \%$ carbon fiber volume fraction.

\section{MATERIAL DESCRIPTION}

A unidirectional carbon fiber-reinforced polymer lamina [8] (Fig. 1) is constructed such that the carbon fibers are coated with carbon nanotubes (CNT) and then cured in epoxy. The schematic in Fig. 1 defines the principal material axes with respect to the fiber direction. Herein, the 1-, 2-, and 3-axes, are referred to as longitudinal, transverse, and outof-plane, respectively. The epoxy was comprised of bisphenol $\mathrm{F}$ resin reacted with epichlorohydrin and cured with triethylenetetramine (TETA). The carbon nanotubes were $(10,10)$ single-walled carbon nanotubes of radius $0.678 \mathrm{~nm}$. It is assumed in the model that there is no chemical bonding between the 3 components: carbon fiber, carbon nanotubes, and the epoxy.
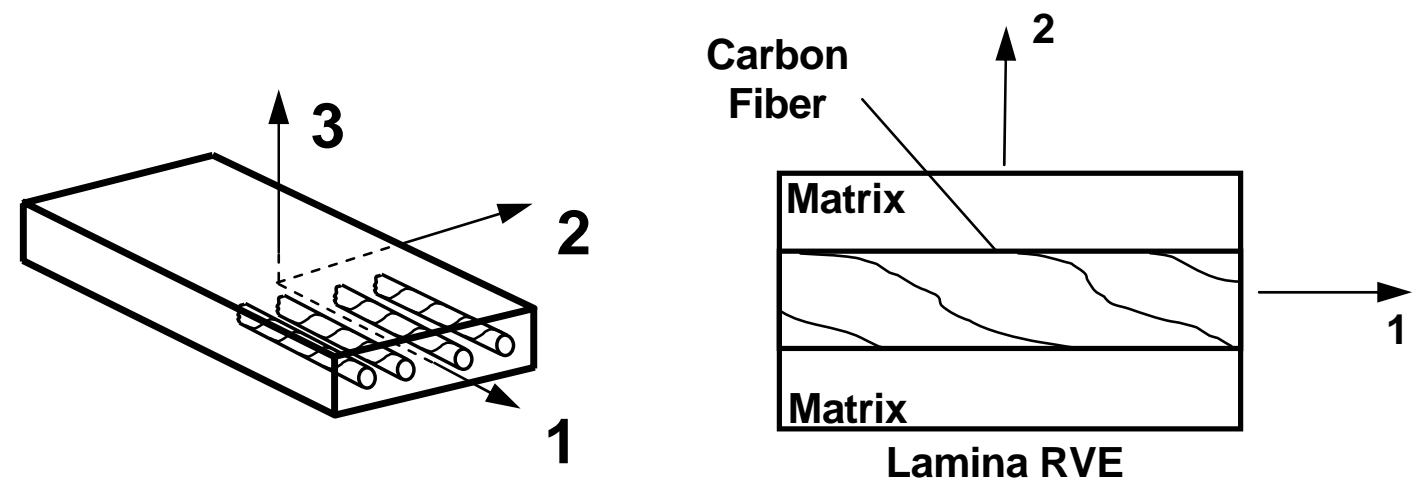

Figure 1. Unidirectionally-reinforced lamina 


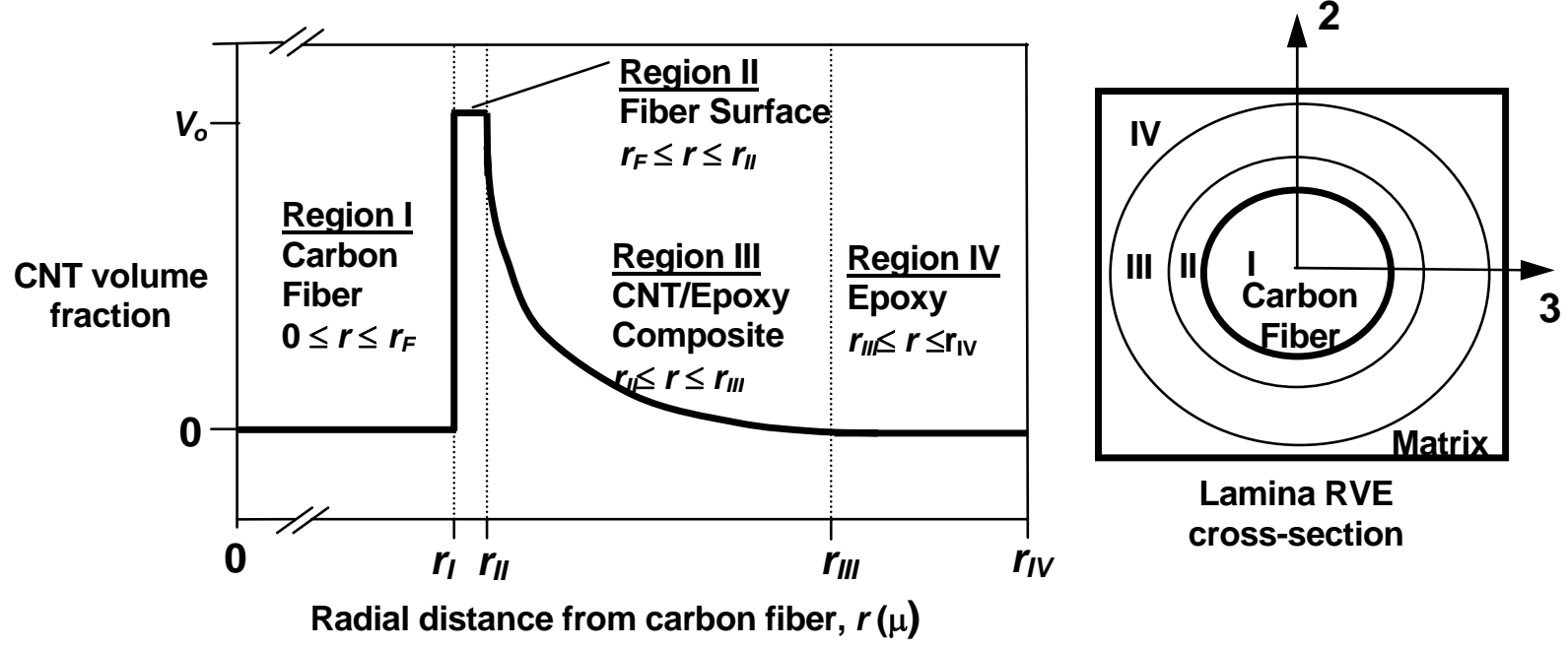

Figure 2. Details of variations of CNT volume fraction in the representative volume element for a lamina with CNT-coated unidirectional carbon fibers

A typical representative volume element (RVE) is shown for a unidirectionallyreinforced lamina in Fig. 1. Details of the variations in the micro-structure resulting from coating the unidirectional carbon fibers with CNT are depicted in Fig. 2, along with details of the lamina RVE cross-section (1-axis, positive out-of-the-page). The carbon fiber and its surroundings are represented by four regions. The CNT volume fraction versus radial distance from the carbon fiber for each region is shown in Fig. 2. Region I is the carbon fiber itself, where $\mathrm{r}_{\mathrm{F}}$ represents the carbon fiber radius. Region II represents the carbon fiber surface, and it has three components: the graphitic surface of the carbon fiber, carbon nanotubes and epoxy, and ranges from the carbon fiber radius at $r_{F}$ to $r_{I I}, 0.004$ microns from the carbon fiber. The CNT volume fraction in Region II is defined by the parameter $V_{o}$, shown in Fig. 2. Region III is the region surrounding the carbon fiber which is comprised of carbon nanotubes and epoxy. As depicted in Fig. 2, the CNT volume fraction in this region decays, from $V_{o}$ to zero, as the radial distance from the carbon fiber increases. The width of Region III ranges from $r_{I I}$ to $r_{\text {III }}$, and is denoted by the parameter $\Delta$, where $\Delta=$ $r_{I I I}-r_{I I}$. Region IV is the remaining epoxy matrix, and ranges from $r_{\text {III }}$ to a maximum width denoted by parameter $\mathrm{r}_{\mathrm{IV}}$.

\section{MULTI-SCALE MODEL}

\section{Molecular dynamics simulations}

Representative volume elements (RVEs) of the molecular structure of the material system were generated via molecular dynamics (MD) simulation. In particular, three different systems with simulated with MD: the epoxy matrix (Fig. 3(a)), the epoxy matrix containing 
(a)

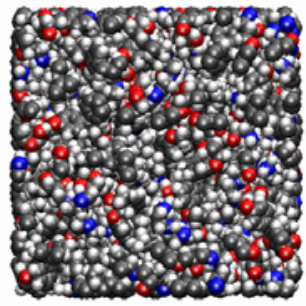

(b)

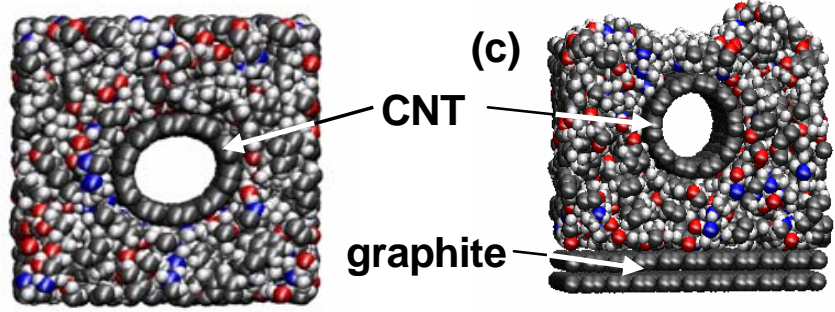

Figure 3. Molecular RVEs of (a) Epoxy, (b) epoxy nanotube composite, and (c) epoxy nanotube and graphite.

a nanotube (Fig. 3b), and the epoxy matrix containing a nanotube, but placed on a graphite surface (Fig 3(c)). The epoxy and the epoxy/nanotube were periodic in all three dimensions, and the three-component system was periodic in the 1- and 2-directions. In the 3-direction was periodic, but extended to 1 micron, and is, therefore, effectively an open boundary.

All three systems were simulated with the AMBER force field [9] using the LAMMPS [10] and DL-POLY [11] simulation codes. All the nanotube and graphite parameters were taken from the AMBER force field. Several of the force field parameters of the epoxy network were derived from $a b$ initio calculations carried out with the NWChem package [12]. The partial atomic charges were derived from an RESP fit to a Hartree-Fock calculation of wavefunction. The bond lengths, angles, and dihedrals, were calculated by optimizing the geometry of parts of the epoxy network at the HF/STO-3G level. Partial charges were then fit at this geometry from a HF/6-31G* calculation. The rest of the parameters, were taken from the AMBER force field. In the simulations, electrostatic interactions were calculated with Coulomb's law, and an Ewald summation was used for the long range Coulombic interactions. Electrostatic interactions and 1-4 non-bonded dihedral interactions were scaled by the standard AMBER parameters of 0.83 and 0.50 respectively.

The molecular structure of the epoxy was a network of bisphenol F resin molecules reacted with epichlorohydrin and cross-linked via triethylenetetramine (TETA) molecules. The TETA molecule is able to cross-link with up to four resin chains, and on average 2.3 of the 4 TETA amine groups in the resultant structure were involved in cross-linking. The equilibrium structures were obtained by compressing the epoxy network until the minimum potential energy configuration at finite temperature $(300 \mathrm{~K})$ was reached. In the 3component system, the epoxy was prepared with periodicity in the 1- and 2- directions, and was compressed between graphite plates. The upper graphite plate was then removed from the simulation, and the system was further equilibrated to minimize the potential energy.

\section{Equivalent-Continuum Model}

The elastic constants were then obtained for each of the molecular RVEs from equating the energies of deformation for the molecular system, which were calculated from molecular dynamics simulation, to the energies of deformation of an equivalent continuum solid under identical deformations. The material constitutive relation of stress $\sigma$ and strain $\varepsilon$ is given by Hooke's law 


$$
\sigma=\mathbf{C} \varepsilon
$$

The stiffness matrix $\mathrm{C}$ of an orthotropic system has 9 components:

$$
\mathbf{C}=\left(\begin{array}{cccccc}
C_{11} & C_{12} & C_{13} & 0 & 0 & 0 \\
C_{12} & C_{22} & C_{23} & 0 & 0 & 0 \\
C_{13} & C_{23} & C_{33} & 0 & 0 & 0 \\
0 & 0 & 0 & G_{23} & 0 & 0 \\
0 & 0 & 0 & 0 & G_{13} & 0 \\
0 & 0 & 0 & 0 & 0 & G_{12}
\end{array}\right)
$$

The 9 components can be determined from the boundary conditions given in Ref. [2]. As in previous work [2], the axial stiffness $\mathrm{C}_{\mathrm{ii}}$ and shear moduli $\mathrm{G}_{\mathrm{ij}}$ were determined directly, and the stiffnesses $\mathrm{C}_{\mathrm{ij}}$ were calculated from the plane strain bulk moduli $\mathrm{K}_{\mathrm{ij}}$ and $\mathrm{C}_{\mathrm{ii}}$. For these cases, $i$ and $j$ are any of the 1-, 2- or 3- principal directions. The $C_{\mathrm{ii}}$ and $\mathrm{K}_{\mathrm{ij}}$ were determined for all three systems. For the epoxy/nanotube system all nine constants were computed. The shear moduli of the remaining two systems were not needed for the model.

In the MD simulations, the displacement fields from Ref. [2] were applied to the each RVE structure in strain increments of 0.0025 every 10,000 steps at 1 fs each (10 ps per increment) up to a maximum strain of greater than 0.02. For the epoxy and epoxy/nanotube systems, application of the displacement field included displacing both the periodic boundaries and the atomic positions, then equilibrating the system with MD to its new state point. For the three-component system, the upper layer of the epoxy and the lower graphite sheet were fixed in location. The atoms in the fixed layers were displaced, but no further equilibration was performed on them.

Because of the limitation of rectilinear boxes in LAMMPS, DL-POLY was used for the shear moduli. Care was taken to translate the force field as accurately as possible between the codes, but some variation in the results is still expected.

\section{Mori-Tanaka Micromechanics}

The elastic constants determined for the molecular RVEs with the molecular dynamics/equivalent continuum method described above, are then used as input to the MoriTanaka micromechanics method to calculate the properties of an embedded epoxy/nanotube effective fiber in an epoxy matrix [13-15]. The stiffness tensor $\mathbf{C}$ of this nanocomposite as a function of effective fiber volume fraction $v_{f}$ is

$$
\mathbf{C}=\left(\left(1-v_{f}\right) \mathbf{C}^{m}+v_{f} \mathbf{C}^{f} \mathbf{T}^{f}\right)\left(\left(1-v_{f}\right) \mathbf{I}+v_{f} \mathbf{T}^{f}\right)^{-1}
$$

where the dilute strain concentration tensor $\mathbf{T}^{f}$ is

$$
\mathbf{T}^{f}=\left[\mathbf{I}+\mathbf{S}^{f}\left(\mathbf{C}^{m}\right)^{-1}\left(\mathbf{C}^{f}-\mathbf{C}^{m}\right)\right]^{-1}
$$


and $\mathbf{S}^{f}$ is the Eshelby tensor for a prolate ellipsoid [16] with aspect ratio 1:1000. The superscripts and subscripts $f$ and $m$ refer to the effective fiber and matrix properties, respectively, and $\mathbf{I}$ is the identity tensor. The $v_{f}$ is related to the nanotube volume fraction $v_{N T}$ as

$$
v_{N T}=v_{N T, R V E} v_{f}
$$

where $v_{N T, R V E}$ is the volume fraction of nanotubes in the molecular RVE. In equation (3) the stiffness matrix $\mathrm{C}$ is calculated for aligned effective fibers. To randomize the orientation of the effective fibers in the nanocomposite[2,17], and therefore the nanotubes, the components of $\mathbf{C}$ are

$$
\left\langle C_{i j m n}\right\rangle=\left(\kappa-\frac{2}{3} \mu\right)\left(\delta_{i j} \delta_{m n}\right)+\mu\left(\delta_{i s} \delta_{j n}+\delta_{i n} \delta_{j m}\right)
$$

where $i, j, m, n=1,2,3, \delta_{i j}$ is the Kronecker delta and

$$
\begin{gathered}
\kappa=\frac{1}{9} C_{i i j j} \\
\mu=\frac{1}{10}\left(C_{i j i j}-\frac{1}{3} C_{i i j j}\right)
\end{gathered}
$$

With the randomization of the effective fibers, $\mathbf{C}$ becomes isotropic.

\section{Distribution of Nanotubes}

The Mori-Tanaka analysis yields the modulus of the nanocomposite at different nanotube loadings, but a description of the spatial distribution of the nanotubes around the carbon fiber must also be defined. The nanotube distribution can be described by using the following exponential form:

$$
v_{d}(r, \alpha)=V_{o} e^{-\alpha\left(r-r_{I I}\right)}-\left(\frac{r-r_{I I}}{\Delta}\right) V_{o} e^{-\alpha \Delta}
$$

where, $v_{d}$ is the volume fraction distribution; the parameter $r$ measures radial distance from the center of the carbon fiber; $V_{o}$ is the initial CNT-epoxy volume fraction of the molecular $\mathrm{RVE}$; and $\Delta$ denotes the width of the region in which the nanotubes are distributed. In the present analysis, a value for $\Delta$ was specified, and the following constraint equation was solved for successive values of the decay parameter $\alpha$ using Mathematica ${ }^{\mathrm{TM}}$ [18] 


$$
\sum_{i}^{N} v_{d}\left(r_{i}, \alpha\right) \int_{r_{i}}^{r_{i+1}} 2 \pi r d r=V_{N} \cdot A .
$$

Here, $V_{N}$ denotes the lamina volume fraction, $A$, the lamina RVE surface area in the 2-3 plane, shown in Fig. 2, and $N$, the number of subregions of Region III. The value of $\alpha$ that satisfies the equality of Eqn (9), for a given value of $V_{N}$, is then applied in the relation of Eqn (8) to define the distribution of CNT volume fractions Region III for width $\Delta$.

\section{Atomistically-Informed Rule-of-Mixtures Analysis}

The contribution to the longitudinal Young's modulus of the lamina, E, from each of the four regions shown in Fig. (2) is then determined by using an atomistically-informed ruleof-mixtures analysis:

$$
E=v_{I} E_{I}+v_{I I} E_{I I}+v_{I I I} E_{I I I}+v_{I V} E_{I V}
$$

where, $v_{i}$ and $E_{i}$ are the volume fraction and Young's modulus of each of the 4 regions. For the present analysis, $v_{I}$ represents the carbon fiber volume fraction, and is fixed at $60 \%$. The Young's modulus of the carbon fiber, $E_{1}$, is $230 \mathrm{GPa}$. For the present analysis, the volume fraction $v_{I I}$ is fixed by setting the value of $r_{I I}$ at 0.004 microns. The Young's modulus $E_{I I}$ is calculated from the $\mathbf{C}$ tensor of the three component molecular RVE, shown in Fig 3c, resulting from the molecular dynamics/equivalent continuum method. The contributions of $v_{3}$ and $E_{3}$ are variable. In Region III the nanotube distribution varies according to Eqns (8) and (9). The variation of the modulus in Region III is taken from the properties derived by mapping the results of the Mori-Tanaka micromechanics model the CNT volume fraction distributions from Eqns (8) and (9). Finally, the Young's modulus and volume fraction for Region IV, $E_{I V}$ and $v_{I V}$ respectively, are calculated from the $\mathbf{C}$ tensor for the epoxy molecular RVE (Fig 3(a)) .

\section{RESULTS AND DISCUSSION}

The elastic constants of the molecular RVEs determined from the molecular dynamics simulations and the equivalent continuum model are listed in Table I. The epoxy elastic constants yield a Young's modulus of $1.44 \mathrm{GPa}$ and a Poisson ratio of 0.42. These values are used for the epoxy matrix in the present analysis. Typical values for storage moduli of neat cured epoxy are 2-3 GPa at 250-300 K [19]. The volume fraction of nanotubes in the epoxy/nanotube system was $15.35 \%$, and in the epoxy/nanotube/graphite system, $15.55 \%$.

Using the nanotube/epoxy composite properties presented in Table I and the properties of the epoxy matrix, the Young's modulus of the nanocomposite with randomized effective epoxy/nanotube fibers is plotted in Fig 4 as a function of nanotube volume fraction. These results are generated using the Mori-Tanaka micromechanics from eqns (3)(7). 
Table I- The Elastic Constants of the Molecular RVEs.

\begin{tabular}{|c|c|c|c|}
\hline $\begin{array}{c}\text { Elastic } \\
\text { Constants }\end{array}$ & $\begin{array}{c}\text { Epoxy } \\
\text { (GPa) }\end{array}$ & $\begin{array}{c}\text { Epoxy/Nanotube } \\
\text { (GPa) }\end{array}$ & $\begin{array}{c}\text { Epoxy/Nanotube/Graphite } \\
\text { (GPa) }\end{array}$ \\
\hline $\mathrm{C}_{11}$ & 4.12 & 99.0 & 267 \\
\hline $\mathrm{C}_{22}$ & 5.05 & 9.43 & 182 \\
\hline $\mathrm{C}_{33}$ & 3.60 & 6.22 & 9.94 \\
\hline $\mathrm{K}_{12}$ & 4.78 & 31.9 & 138 \\
\hline $\mathrm{K}_{13}$ & 4.73 & 32.4 & 72.2 \\
\hline $\mathrm{K}_{23}$ & 4.41 & 12.1 & 49.5 \\
\hline $\mathrm{G}_{12}$ & & 0.33 & \\
\hline $\mathrm{G}_{13}$ & & 0.32 & \\
\hline $\mathrm{G}_{23}$ & & 0.20 & \\
\hline
\end{tabular}

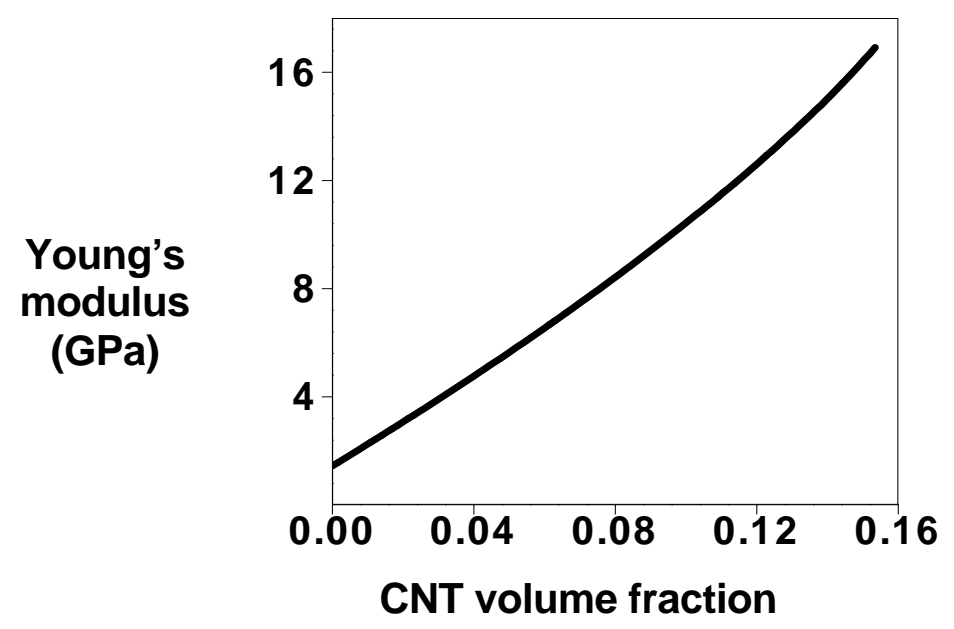

Figure 4. Young's modulus of the epoxy/CNT composite from Mori-Tanaka micromechanics

Fig 5(a) shows variations in CNT volume fraction distributions of exponential form for a lamina RVE with $60 \%$ carbon fiber volume fraction and $\Delta=0.3$ microns. Here, volume fraction variations from Eqns (8) and (9) are depicted for lamina CNT volume fractions ranging from 0.0005 to 0.0055 . The exponential form allows the CNT volume fraction distribution to vary from a nearly linear form as shown for $V_{N}=0.0055$ through increasingly exponentially varying forms for decreasing values of lamina CNT volume fraction, to a nearly stepwise variation at $V_{N}=0.0005$.

Fig 5(b) shows the variations in Young's modulus for Region III. Results are shown for selected nanotube distributions from Fig 5(a). Here, Mori-Tanaka micromechanics have been applied using volume fraction distributions for lamina volume fractions 0.0055 , 0.0025, and 0.0005, where $\Delta=0.3$ microns. The Young's modulus distributions of Fig 5(b) are used directly in the atomistically-informed rule-of-mixtures analysis to represent the contribution of Region III to the constitutive properties of the lamina. 

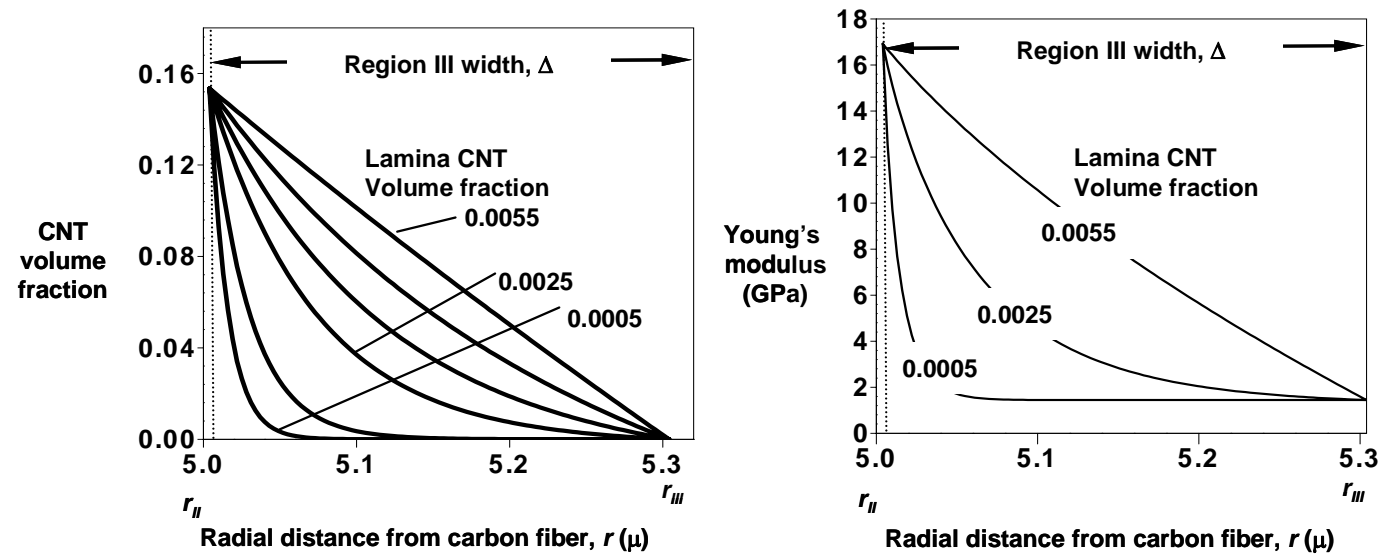

Figure 5. (a) CNT volume fraction distribution in Region III and (b) Young's modulus distribution in Region III, for $\Delta=0.3$.

Finally, the total longitudinal Young's modulus of the unidirectionally-reinforced lamina with CNT is plotted in Fig. 6 for different sizes of Region III (Fig. 2). For nanotubes within approximately $700 \mathrm{~nm}$ of the carbon fiber surface the Young's modulus varies about $0.01 \mathrm{GPa}$ with changes to the size of Region III. The predominant change in the Young's modulus of the coated nanotube fiber is a function of the nanotube volume fraction in the lamina. Up to nanotube loadings of 0.0125 , the amount of change in the modulus is 1.2 GPa.

\section{CONCLUSIONS}

A multi-scale method, denoted the atomistically-informed rule-of-mixtures, is developed to calculate the Young's modulus of a carbon nanotube coated carbon fiber.

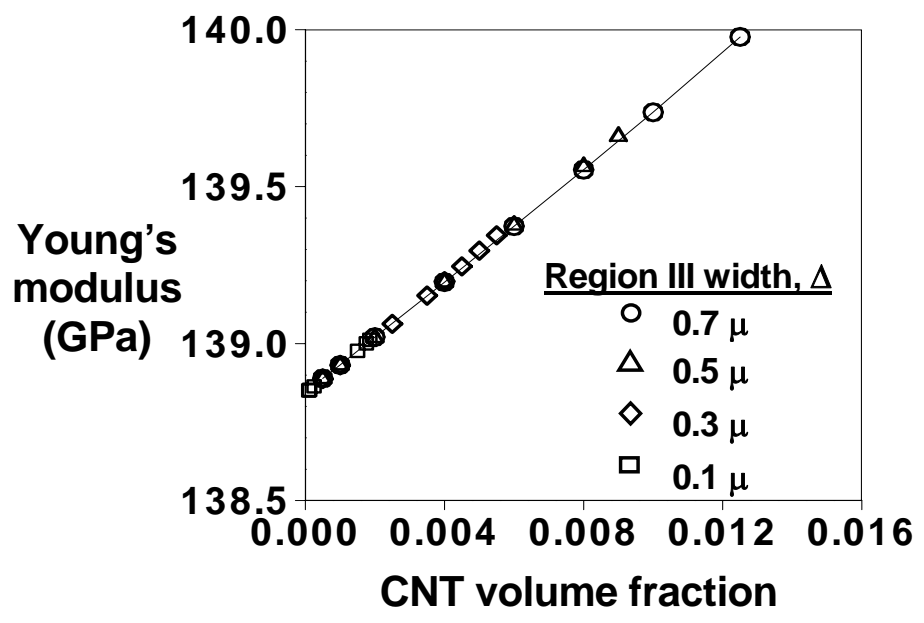

Figure 6. Atomistically informed rule-of-mixtures results for unidirectionally-reinforced lamina with CNTcoated carbon fibers. 
Within the method, the distribution of nanotubes in the vicinity of the carbon fiber surface can be varied as a function of nanotube volume fraction. For a lamina with $60 \%$ carbon fiber volume fraction, the results indicate that the Young's modulus is less dependent on the local nanotube disribution than on the nanotube volume fraction for nanotube located within $700 \mathrm{~nm}$ of the carbon fiber and nanotube loadings in the lamina of up to $1.25 \mathrm{vol} \%$.

\section{ACKNOWLEDGMENTS}

The authors thank D. C. Lagoudas, G. D. Seidel, J. Zhu, E. Barrera, P. Thakre, A. Awasthi, and J. Ratcliffe for many helpful discussions. The simulations were carried out at the NASA Ames Supercomputing Center, and on System X at Virginia Polytechnic Institute and State University.

\section{REFERENCES}

1. X. L. Gao and S. Mall, J Comp Tech Res 22, (2000)

2. S. J. V. Frankland, G. M. Odegard, and T. S. Gates, AIAA Journal 43, 1828-1835 (2005).

3. T.S. Gates, G.M. Odegard, S.J.V. Frankland and T.C. Clancy, Composite Science and Technology 65, 2416 (2005).

4. G. M. Odegard, S. J. V. Frankland, M. N. Herzog, T. S. Gates, and C. C. Fay. "Constitutive Modeling of Cross-Linked Nanotube Materials” 45th AIAA/ASME/ASCE/AHS/ASC Structures,Structural Dynamics, and Materials Conference, AIAA-2004-1606.

5. T. S. Gates, G. M. Odegard, M. P. Nemeth, and S. J. V. Frankland. "Predicting the Influence of Nano-scale Material Structure on the In-plane Buckling of Orthotropic Plates" 45th AIAA/ASME/ASCE/AHS/ASC Structures, Structural Dynamics, and Materials Conference, AIAA-2004-1607.

6. J. Gou, Z. Liang, C. Zhang, and B. Wang, Composites B 36, 524-533 (2005).

7. T. C. Clancy, and T. S. Gates "Mechanical Properties of Nanostructured Materials Determined through Molecular Modeling Techniques”, 46th AIAA/ASME/ASCE/AHS/ACS SDM Conference, AIAA2005-1852.

8. R. M. Jones, Mechanics of Composites Materials, (Taylor and Francis, 1975).

9. W. D. Cornell, P. Cieplak, C.I. Bayly, I.R. Gould, K.M. Merz, D.M. Ferguson, D.C. Spellmeyer, T. Fox, J.W. Caldwell and P.A. Kollman. J. Am. Chem. Soc. 117, 5179 (1995).

10. S. J. Plimpton, J. Comp. Phys. 117, 1 (1995).

11. W. Smith and T. R. Forester, DL-POLY, Daresbury, Nr. Warrington, England: The Council for the Central Laboratory of the Research Councils, 1996.

12. High Performance Computational Chemistry Group. "NWChem, A Computational Chemistry Package for Parallel Computers, Version 4.5" Pacific Northwest National Laboratory, Richland, WA, 2003.

13. T. Mori and K. Tanaka, Acta Metallurgica 21, 571 (1973).

14. Y. Benveniste, Mech. Mat. 6, 147 (1987).

15. G. M. Odegard, S. J. V. Frankland, and T. S. Gates, "The Effect of Chemical Functionalization on Mechanical Properties of Nanotube/Polymer Composites”, $44^{\text {th }}$ AIAA/ASME/ASCE/AHS/ASC Structures, Structural Dynamics, and Materials Conference, AIAA-2003-1701.

16. R.M. Christensen, Mechanics of Composite Materials (John Wiley \& Sons, New York, 1979). p. 95.

17. J. G. Berryman, J. Acoust. Soc. Am. 68, 1820 (1980).

18 . S. Wolfram, The Mathematica Book, 4th Ed, (Wolfram Media, Cambridge, MA, 1999).

19. D. H. Kaeble, J. Moacanin, and A. Gupta "Physical and Mechanical Properties of Cured Resins”, in Epoxy Resins: Chemistry and Technology, 2nd edition, C. A. May, ed. (NewYork: Marcel Dekker, Inc., 1988) pp. 603-651. 